\title{
Modelling collective foraging by means of individual behaviour rules in honey-bees
}

\begin{abstract}
An individual-oriented model is constructed which simulates the collective foraging behaviour of a colony of honey-bees, Apis mellifera. Each bee follows the same set of behavioural rules. Each rule consists of a set of conditions followed by the behavioural act to be performed if the conditions are fulfilled. The set of conditions comprises the state of external information available to the bee (e.g. the dancing of other bees) and internal information variables (like memorised location of a food source and homing motivation). The rules are partly observational (i.e. they capture the observable regularities between the present external information and the individual bee's behaviour), and partly involve hypothesised internal-state variables (e.g. abandoning tendency and homing motivation), because no observable (physiological) aspect has as yet been detected in the bee which correlates with changes in the internal motivation. Our aim is to obtain a set of rules that is necessary and sufficient for the generation of the collective foraging behaviour observed in real bees. We simulated an experiment performed by Seeley et al. in which a colony of honey-bees chooses between two nectar sources of different profitabilities which are switched at intervals. A good fit between observed and simulated collective forager patterns was obtained when the model included rules in which the bees (1) relied on the information acquired from previous flights to a source (e.g. profitability and time of day when the source was found), (2) used positional information obtained by attending recruitment dances and (3) did not abandon a (temporarily) deteriorated source too fast or too slowly. The significance of the following issues is discussed: the role of internal and external information, source profitability, the spatial precision of the dance communica-
\end{abstract}

Han de Vries $(\bowtie) \cdot$ Jacobus C. Biesmeijer

Ethology and Socio-Ecology Group, Utrecht University,

P.O. Box 80.086, 3508 TB Utrecht, The Netherlands

e-mail: J.deVries@bio.uu.nl, Tel.: +31-30-2535403,

Fax: + 31-30-2521105 tion, the ability to search for a source after the source position has been transmitted, the tendency to abandon a deteriorated source, and the concepts of scout, recruit, (un)employed forager, and foraging history.

Key words Honey-bee Collective behavior Collective foraging $\cdot$ Communication $\cdot$ Individual-oriented model

"It may be that. You never can tell with bees." from Winnie the Pooh by A.A. Milne (1926).

\section{Introduction}

A colony of honey-bees has to acquire nectar (and pollen) from the flowers in the vicinity of the nest. Since the availability of the nectar sources varies in space and time, the colony of bees has to adapt itself continually to each new situation if it is to procure the necessary amount of nectar. An appropriate division of the worker force between exploring the field for new rich sources and exploiting the known sources is obviously important for the maintenance of the colony. Many studies (reviewed by von Frisch 1967; Gould and Gould 1988; Seeley 1995) have investigated how the foraging behaviour of each individual bee is regulated and what types of external information (such as odour, location information in the waggle dance, the presence of other bees at the source or between the hive and the source) and internal information (such as remembered source location or source odour) play significant roles in this foraging behaviour.

Food source communication: the waggle dance

It has been known for a long time that foraging honeybees provide information to other bees in the hive about the location of the food source they have visited. An important means for doing this is the waggle dance, 
which consists of a series of waggle runs, each followed by a semi-circular return. The duration of the waggle run, the central portion of the dance, is correlated with the distance to the food source. The direction of the waggle run relative to the gravitational axis is correlated with the direction relative to the sun's position in which the food source is to be found (von Frisch 1967; Seeley 1995, pp. 36-38). Information about both distance and direction is used by dance-following bees in their search for the advertised source (Michelsen et al. 1992). The total duration of the dance is correlated with the profitability of the source, but this information is not used as such by the bees attending the dance. There are some other features of the dance that also correlate with the profitability of the source, such as the rate of reversals and several aspects of the dance sounds, which make the dance look fairly vigorous to the human observer (Waddington and Kirchner 1992). The bees that follow the dancers appear to sample a dance at random (Seeley 1995, p. 152; Seeley and Towne 1992) and do not compare different dances with each other with respect to profitability. However, the more vigorous and longer a dance, the more followers it will probably attract.

We are aware that the distance and direction to the food source encoded in the waggle dance are not the only types of information that may guide searching bees to profitable food sources. Odour cues both in the hive (von Frisch 1923; Wenner and Wells 1990) and in the field (Friesen 1973; Wenner and Wells 1990) and visual stimuli (Wenner and Wells 1990) all influence the behaviour of searching bees. Because many empirical studies deal specifically with the honey-bee's dance behaviour and its function (von Frisch 1967; Seeley 1995) as do theoretical studies (Camazine and Sneyd 1991; Bartholdi et al. 1993), our modelling exercise concentrated on honey-bee communication of a food source by means of the waggle dance.

An individual-oriented model

of honey-bee nectar foraging

In this paper we develop an individual-oriented (i-o) simulation model of a colony of bees foraging on two nectar sources of which the sugar concentrations (the profitabilities) are varied over time. Each bee is represented as an independent individual whose behaviour is regulated by a behaviour control structure. At each moment, a bee's behaviour is determined by the internal and external information available to it and its motivational state, according to a set of specific rules. The set of rules is identical for each bee, but since the perceptible environment differs for bees with a different spatial location, the behaviour differs too. Bees can also show different behaviour because of differences in their foraging experience and/or their motivational state.

An important goal of our study is to demonstrate the advantages of achieving a close coupling between an i-o simulation model and the acquisition of empirical data.
We can only record a limited number of observations from which an empirical rule can be derived. By integrating such rules into an i-o simulation model and observing the behaviour of the model, one has the opportunity to examine the sufficiency and necessity of these rules. The validity of the model as a whole depends on the empirical validity of each of the behavioural rules.

We started our modelling exercise with a modest goal: we simply wanted to construct a model that would generate the collective foraging patterns observed by Seeley et al. (Fig. 2 in Seeley et al. 1991). We wanted to use an i-o simulation model rather than a mathematical model involving a system of non-linear differential equations as developed by Camazine and Sneyd (1991). An important criterion for using i-o simulation modelling is that empirical rules can be easily incorporated into the model, thus providing a more direct link between observation and modelling. We believe that this is more difficult with differential-equation modelling. Of course, different modelling formalisms have different advantages and disadvantages. In fact they can often complement each other. See Huston et al. (1988), Hogeweg and Hesper (1990), Villa (1992) and Judson (1994) for reviews of modelling formalisms with emphasis on the i-o (or individual-based) modelling approach.

The main objective: what rules do foraging honey-bees use?

The main goal of this modelling study is to find the rules, followed by each individual bee, which are necessary and sufficient to explain the collective foraging behaviour. These rules must specify for each bee when, how long and where the bee will perform some type of behaviour. These rules include specifications for: (1) the travelling of an outgoing bee from the nest to the source, (2) searching for the source by a bee, (3) the collecting of nectar from a source, (4) travelling back to the nest, (5) the way in which information about the source is transmitted to other bees in the nest, that is, the dancing of the returning bee, (6) the reaction of a bee in the nest to the dancing of a nest mate. It is obviously essential that bees have a memory, so rules have to be drawn up which determine what type of information sensed in the environment will be recorded in a bee's memory for later use. Additionally, there must be rules about forgetting memorised features when new information becomes available to the bee in the perceptible part of the environment. Also, rules about making errors (in dancing or in travelling) are to be included. We devised these rules on the basis of the knowledge that is available about honey-bee foraging. If a necessary rule or value of a parameter could not be extracted from the literature we chose one that appeared to be likely. When this happens in the modelling exercise it is a clear instigation for further empirical research. We consider this a positive feature of i-o simulation modelling. We think that in this 
way the rate at which integrated knowledge of the subject is acquired will be high.

The structure of this paper

In the next section, we present the model specifying the rules and the parameters. This is followed by the results of experimenting with this simulation model. We then discuss the types of empirical questions that were raised while we were developing and experimenting with the model. We also discuss in what way the i-o modelling process may help with the construction of an explanatory theory. The paper ends with suggestions for future extensions of the model.

\section{Modelling and simulation}

We developed an i-o model as a SMALLTALK simulation program using the Hobo event-driven simulation system developed by Lhotka (1994). As a simulation test-bed we used the feeding experiment designed by Seeley et al. (1991). Each of two nectar sources are placed $400 \mathrm{~m}$ away from the hive. In the original experiment, 12 bees were trained to feed at the north feeder, 15 other bees at the south feeder. In our simulation runs, we used the same numbers of bees with foraging experience of the north and south feeder. The sugar concentrations of the two sources were varied according to the regime given in Table 1. Seeley (1995, p. 142) remarks that the north feeder was provided with a $1.0 \mathrm{M}$ instead of a $0.75 \mathrm{M}$ sugar solution in the morning to prevent total abandonment of this feeder.

The model bee
- time Of Day Found

- position (distance from hive; direction from hive)

- profitability (= concentration)

- (odour)

- (colour)

motivational properties (states):

- homing Motivation (varies between 0 and 1)

- foraging Motivation ( 0 or 1$)$

- abandoning Tendency (varies between 0 and 1 )

The model environment

The space in which the bees move around is a square area of 1200 by $1200 \mathrm{~m}$. In the middle is the hive and there are two sources, one $400 \mathrm{~m}$ south of the hive and the other $400 \mathrm{~m}$ north. Since both sources are at the same distance from the nest, profitability equals the sugar concentration of the source. Bees are restricted so that they can fly around only within $500 \mathrm{~m}$ from the hive. If a bee happens to cross the $500 \mathrm{~m}$ border, her direction of movement is set to point in the direction of the hive.

The only part of the real hive that is included in the model is the dance floor (cf. Seeley 1994, pp. 57-58). This is modelled as a circular area with a radius of $25 \mathrm{~cm}$. The distance from which a bee can perceive (hear) a dancing bee is set to $5 \mathrm{~cm}$ (Michelsen et al. 1987, 1992). So on average, each bee can perceive $5^{2}$ $25^{2}=4 \%$ of the bees on the dance floor at any moment in time. All bees have a random position on the dance floor and therefore the chance of perceiving a dancing bee is independent of the spatial distribution of the bees. This results in dancing bees being randomly sampled and followed by other bees (Seeley and Towne 1992; Seeley 1995).

The parameters (input parameters for the model)

Note: the standard is the value that rendered the best fit of the model.

The number of bees, i.e. the foraging workforce $(N)$ (settings: 50, 100, 150, 200, 250; standard: 200)

We assumed that a fixed number of bees of all possible worker bees present in the colony had a basic propensity for foraging behaviour. In our model, we therefore only simulate the behaviour of these potential foragers.
Table 1 Experimental regime of sucrose concentration at the feeders (after Seeley et al. 1991)

\begin{tabular}{llllll}
\hline & \multicolumn{2}{l}{ Day 1 (19 June) } & & & Day 2 (20 June) \\
\cline { 2 - 3 } \cline { 5 - 6 } & $\begin{array}{l}0800-1200 \\
\text { hours }\end{array}$ & $\begin{array}{l}1200-1600 \\
\text { hours }\end{array}$ & & $0800-1200$ hours & $\begin{array}{c}1200-1600 \\
\text { hours }\end{array}$ \\
\hline North feeder: & $1.00 \mathrm{M}$ & $2.50 \mathrm{M}$ & & $2.50 \mathrm{M}$ & $0.75 \mathrm{M}$ \\
South feeder: & $2.50 \mathrm{M}$ & $0.75 \mathrm{M}$ & & $0.75 \mathrm{M}$ & $2.50 \mathrm{M}$ \\
\hline
\end{tabular}


The transmission accuracy $(t)$ (settings: range 0-1; standard: 0.85 )

This parameter controls the accuracy of the transmission of the source position that is coded in the waggle dance performed by a returning bee. A dancing bee communicates to other bees attending her dance the position (distance and direction) of the source she has just visited. The higher the transmission accuracy, the more accurately the source position is transmitted. This transmission accuracy parameter combines a possible error in the information present in the dance ("writing error") with a possible error made during "reading" distance and direction from the dance ("reading error"). With the accuracy set to 1 , the source position is communicated without error. When the transmission accuracy is set to 0.8 , a source position is communicated from the dancer to the follower with an error drawn randomly from a uniform distribution between 0 and $200 \mathrm{~m}$. If $t=0.5$, the random error is between 0 and $500 \mathrm{~m} ; t=0$ corresponds to a random error between 0 and $1000 \mathrm{~m}$. Experiments performed by Towne and Gould (1988, Fig. 8) show that most recruits search within about $200 \mathrm{~m}$ from the target source for a target located at $450 \mathrm{~m}$ from the hive.

The search ability $(s)$ (settings: 2, 3, 4, 8, 16; standard: 4)

The search ability controls how accurately a source can be located on the basis of the approximate source position read from a waggle dance. A maximum value of $s$ means that the registered source is found by the searching bee in an optimal way. A lower value of $s$ means that the bee takes a more erratic route towards the memorised source and that at the same time the memorised source position is subject to random changes. With a standard value of 4 for $s$ (and the standard value of 0.85 for the transmission accuracy) almost all recruited bees will search within $200 \mathrm{~m}$ of the target source (cf. Towne and Gould 1988) and on average a bee will make about five unsuccessful search flights before finding the target (cf. Seeley 1995, pp. 126-127). Seeley (1983, p. 257) reports that recruits required $4.8 \pm 3.2$ (range 1-12) dance-guided search trips to find a target after having followed a dance.

The abandoning probability (a) (settings: range 0-0.20; standard: 0.07)

This parameter is the probability per flight trip that the bee will abandon a source having a sugar concentration of $0.75 \mathrm{M}$. The tendency to abandon a source depends not only on the value of this abandoning probability parameter but also on the profitability of the source just visited. In order to take this into account, the abandoning probability for the $1.0 \mathrm{M}$ source is always set at a value 0.15 lower (with a minimum value of 0 ) than the abandoning probability of the $0.75 \mathrm{M}$ source (cf. Seeley et al. 1991, table 1). Note that the $2.5 \mathrm{M}$ source is never abandoned.

A bee exploiting a rich source (the $2.5 \mathrm{M}$ source) will become unemployed when this source has deteriorated. Since this bee has foraging experience with this particular source she will not be inclined to follow recruitment dances right away. It will be some time before she shows interest in other dances again (cf. Seeley 1995, p. 125) The duration of this transitional phase is controlled by the abandoning probability parameter. So this parameter controls how fast a bee's tendency to abandon a rich source increases after the sucrose concentration of this source has become $0.75 \mathrm{M}$. It also controls the bee's tendency to attend dances again after becoming unemployed. As soon as her abandoning tendency has reached its maximum she will begin to follow dances again. At this point she has fully abandoned her source.

\section{Terminology}

To describe a bee's foraging career we will use the following terminology which we first outline and then describe in more detail (cf. Seeley 1995, pp. 85-88, 122). Since more and more is becoming known about the behaviours performed by a forager bee and how they are controlled by internal and external information, the original scout-recruit concept (e.g. Seeley 1983) is being refined and extended. The categories of foragers used in this paper are as follows:

employed forager (knows and exploits a profitable source; does not follow dances)

unemployed forager (is not exploiting a source)

- naive (or novice) forager

- scout (starts searching spontaneously; does not know a source)

- recruit (starts searching upon attending a dance; knows approximately the position of a source but not its profitability)

experienced forager (knows position and profitability of a source)

- inspector (reactivates spontaneously to make a reconnaissance visit to inspect the profitability status of the source)

- reactivated forager (is reactivated upon attending a dance that contains confirming information)

- scout (starts searching spontaneously for some new, unknown source after her own source has deteriorated)

- recruit (starts searching a new, advertised source upon attending a dance that contains information which does not agree with her known source)

A potential forager bee starts her career as an unemployed naive worker, that is, she has as yet no knowledge of a food source in the field. She can start searching for a source "spontaneously" and thus become a scout (explorer). "Spontaneously" here means 
that the instigation to fly out and start foraging is not due to following a waggle dance but due to some unknown internal, motivational factor or possibly to some as yet unknown external cue. Alternatively, a bee can start searching for a source as a response to attending a waggle dance and thus become a recruit. So the difference between a recruit and a scout is that the former has stored approximate positional information in her memory, whereas the latter has not.

As soon as a bee finds a source, she registers the particulars of this source in her memory and starts exploiting it; the bee is then an employed forager (exploiter). An employed forager can become unemployed if she stops (permanently or temporarily) exploiting her source because the source has been (temporarily) exhausted. As long as she keeps the information about this source in her memory (which can be inferred from seeing her making a reconnaissance flight to this source), this bee is called an experienced, unemployed bee. When such a bee makes a reconnaissance flight to her known source she will be called an inspector. If such a bee attends a dance which contains information that fits the particulars of her known source, and is thereby stimulated to go out to her source she will be called a reactivated forager. [Note that the dancing bee may in fact advertise a different source from the one which the
Fig. 1 Some of the behavioural rules used in the model. Notes: (a) The probability to fly out is determined by both the known source concentration and the abandoning tendency. If the known source concentration is low but the abandoning tendency is still weak, the bee will make (once in a while) a reconnaissance flight. (b) The error in the communicated source position is inversely related to the transmission accuracy. (c) During the search for a source, the approximate position of which has been obtained from a dancing bee, the following takes place. Slowly, the memorised source position changes randomly. The higher the search ability, the smaller the random error added to the approximate source position. Homing motivation increases slowly. After the bee has travelled more than $2000 \mathrm{~m}$, the homing motivation has become so high that the bee returns to the nest. So, within about 16 min, a bee will return from an unsuccessful search trip [see Seeley and Towne 1992, p. 64: "Typically, the bee made several $(4.1 \pm 2.7,1-10$, $\mathrm{n}=21$ ) of these dance-guided searches, with each unsuccessful search lasting about a quarter of an hour $(16 \pm 8.3,2-$ 37 min, $n=64)$."']. These bees searched for flower patches between 150 and $3000 \mathrm{~m}$ from the hive. See also Seeley (1983, p. 257): "Recruits did not find the target patch on their first trip out after following a dancer [who advertised a target at $60 \mathrm{~m}$ from the hive]. Instead, they required $4.8 \pm 3.2$ (range $1-12$ ) dance-guided search trips, each one lasting $17 \pm 11 \mathrm{~min}$ "

\section{IN NEST:}

IF bee carries load THEN

1.unload (time increases with increasing source prof) (Seeley et al. 1991, table 1)

2.dance (time linearly related to source prof) (Seeley 1995:98; Seeley et al. 1991, table 1)

3.delay (time decreases with increasing source prof) (Seeley et al. 1991, table 1).

IF bee does not carry load THEN

\section{FLY OUT OR STAY IN:}

IF bee is naive (doesn't know a source) THEN stay in.

IF bee is experienced (knows a source) THEN

IF the known source concentration is high enough AND the tendency to abandon is weak enough THEN fly out to the known source (a)

ELSE stay in, i.e. this bee becomes unemployed, but retains source info in her memory (she remains experienced).

2. DELAY:

FOR all bees: delay for some random time (uniform between 2 and 5 minutes).

\section{FOLLOW DANCE OR NOT:}

IF bee is naive (doesn't know a source) THEN

IF a dancing bee is perceived THEN

source position of dancing bee is transmitted (b);

fly out and start searching for the communicated source (bee becomes recruit).

ELSE stay in and go to 2. DELAY

IF bee is experienced THEN

IF a dancing bee is perceived THEN

IF bee has a weak tendency to abandon her source THEN ignore the dance (no action is undertaken).

IF bee has a strong tendency to abandon her source THEN IF the advertised source agrees with her known source THEN fly out to the known source

ELSE stay in and increase tendency to abandon known source.

IF bee has a maximum tendency to abandon her source THEN source position of dancing bee is transmitted (b); fly out and start searching for the communicated source

ELSE go to 1. FLY OUT OR STAY IN

\section{OUTSIDE NEST:}

IF homing motivation is maximum THEN return to nest

ELSE search for source (c).

\section{AT SOURCE:}

1. collect load (time decreases with increasing source profitability)

2. record the source position

3. set homing motivation to maximum

4. adjust the bee's tendency to abandon the source depending on the source concentration (high source conc $=>$ decrease abandoning tendency) (low source conc $=>$ increase abandoning tendency) 
reactivated bee reads from her dance. This depends on how accurately the information in the dance is transferred and on the kind(s) of information the follower bee extracts from the dance.] If an experienced, unemployed bee attends a waggle dance which communicates a source that is unknown to her, she can be stimulated to start searching this unknown source, in which case she will be called a recruit. Alternatively, she might also start scouting again, that is she might start searching for some new unknown source spontaneously after her own source has been depleted and if she does not find any dances to follow on the dance floor. Scout bees are not included in our model since little is known about their searching behaviour (Seeley 1995, p. 87). If their function is to detect good sources not yet known to the colony, then their behaviour will not alter the colony's collective forager pattern given the experimental design used in this model, since the only two sources available are the ones that are already known to the colony. Moreover, the chance that a scout would find one of these two sources by random search would be very small, so the effect of including scouts in the model would only be that the number of unemployed bees inside the nest is somewhat diminished.

The model bee rules

The behavioural rules that are used in the model are presented in Fig.1 and the corresponding behav- iour control structure of the model bee is depicted in Fig. 2.

\section{Model 1: simulating one day}

Our first step was to develop an i-o model which would generate the colony's forager pattern on one day as observed and modelled by Seeley et al. (1991, Fig. 2, 19 June; reproduced as Fig. 3A here). The sugar concentrations of the two feeders (a north and a south feeder) were set according to Table 1 (19 June data). Using the above-mentioned standard parameter setting $(t=0.85$, $s=4, a=0.07$ ), we obtained a forager pattern which agreed well with the observed pattern. Initially however, one feature in our model outcome deviated from the observed pattern. The number of different bees visiting the south feeder in the first half hour after the switch (1200-1230 hours) was always at least as high as in the half hour before the switch, whereas in reality, a strong decrease was observed. This necessitated the introduction of an extra rule into our initial model. We opted for the following rule: a bee which has visited a source once will not always find this source the next time she flies out. The more visits paid to the source the easier it is for her to find this source. With this extra rule, the model outcome agreed with the observed data. This is discussed in the Results.

As the main output parameter we used the number of different bees that visited each of the two sources during
Fig. 2 The behaviour control structure of a forager bee

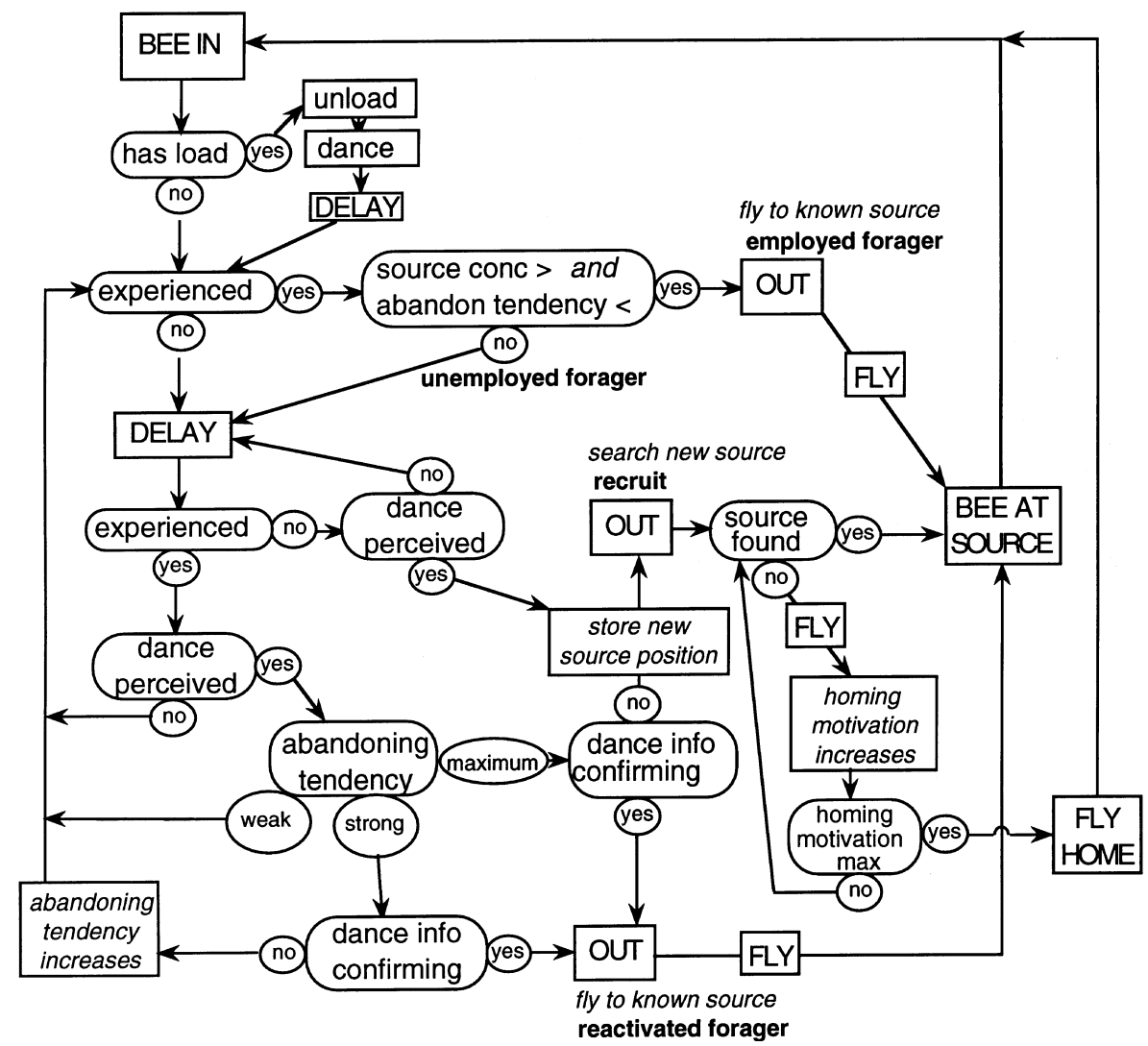


consecutive 30-min periods, which we will call the forager pattern. The following parameters were varied to investigate in what way the forager pattern depends on the values of these parameters.

- Transmission accuracy (range: 0.67-1.0)

- Search ability (range: 2-16)

- Abandoning probability (range: $0.0-0.20$ )

\section{Model 2: simulating two consecutive days}

When we simulated two consecutive days with the model described above we were unable to reproduce the empirical results presented by Seeley et al. (1991). Therefore, the model was extended with an extra feature: a bee memorises the time of the day at which she visits the source for the first time. This feature proved necessary in order to regain the pattern of foraging visitors found on the second day by Seeley et al. This is discussed more fully in the Results.

This two-day model was used to investigate the dependence of the forager pattern on the size of the worker force and on the profitabilities of the sources. To this end we made simulation runs with different numbers of bees $(50,100,150,200$ and 250). In addition, we varied the profitabilities (i.e. the sugar concentration) of the two sources according to three regimes presented in Table 2, using a forager workforce size of 200 bees for each of these simulation runs.

\section{Results}

Although it was fairly simple to develop the basic form of the i-o model of collective foraging, testing and re-
Table 2 Settings for runs in which the source profitabilities were varied

Fig. 3 A Forager pattern of a honey-bee colony visiting two feeders (north and south) both at $400 \mathrm{~m}$ distance from the hive, after Seeley et al. (1991). The values indicate the number of foragers visiting each of the two sources during each half hour. Each value denotes the number of different individuals that visited each source during the previous half hour. For further details see text. B The forager pattern resulting from our i-o simulation model $(t=0.85$, $s=4, a=0.07$ )

\begin{tabular}{|c|c|c|c|c|c|}
\hline & & \multicolumn{2}{|l|}{ Day 1} & \multicolumn{2}{|l|}{ Day 2} \\
\hline & & $\begin{array}{l}0800-1200 \\
\text { hours }\end{array}$ & $\begin{array}{l}1200-1600 \\
\text { hours }\end{array}$ & $\begin{array}{l}0800-1200 \\
\text { hours }\end{array}$ & $\begin{array}{l}1200-1600 \\
\text { hours }\end{array}$ \\
\hline Standard & $\begin{array}{l}\text { North source } \\
\text { South source }\end{array}$ & $\begin{array}{l}1.00 \mathrm{M} \\
2.50 \mathrm{M}\end{array}$ & $\begin{array}{l}2.50 \mathrm{M} \\
0.75 \mathrm{M}\end{array}$ & $\begin{array}{l}2.50 \mathrm{M} \\
0.75 \mathrm{M}\end{array}$ & $\begin{array}{l}0.75 \mathrm{M} \\
2.50 \mathrm{M}\end{array}$ \\
\hline \multirow{2}{*}{$\begin{array}{l}1.5 \mathrm{M}=\text { higher } \\
\text { concentration }\end{array}$} & North source & $1.00 \mathrm{M}$ & $1.50 \mathrm{M}$ & $1.50 \mathrm{M}$ & $0.75 \mathrm{M}$ \\
\hline & South source & $1.50 \mathrm{M}$ & $0.75 \mathrm{M}$ & $0.75 \mathrm{M}$ & $1.50 \mathrm{M}$ \\
\hline \multirow{2}{*}{$\begin{array}{l}1.5 \mathrm{M}=\text { lower } \\
\text { concentration }\end{array}$} & North source & $1.50 \mathrm{M}$ & $2.50 \mathrm{M}$ & $2.50 \mathrm{M}$ & $1.50 \mathrm{M}$ \\
\hline & South source & $2.50 \mathrm{M}$ & $1.50 \mathrm{M}$ & $1.50 \mathrm{M}$ & $2.50 \mathrm{M}$ \\
\hline
\end{tabular}

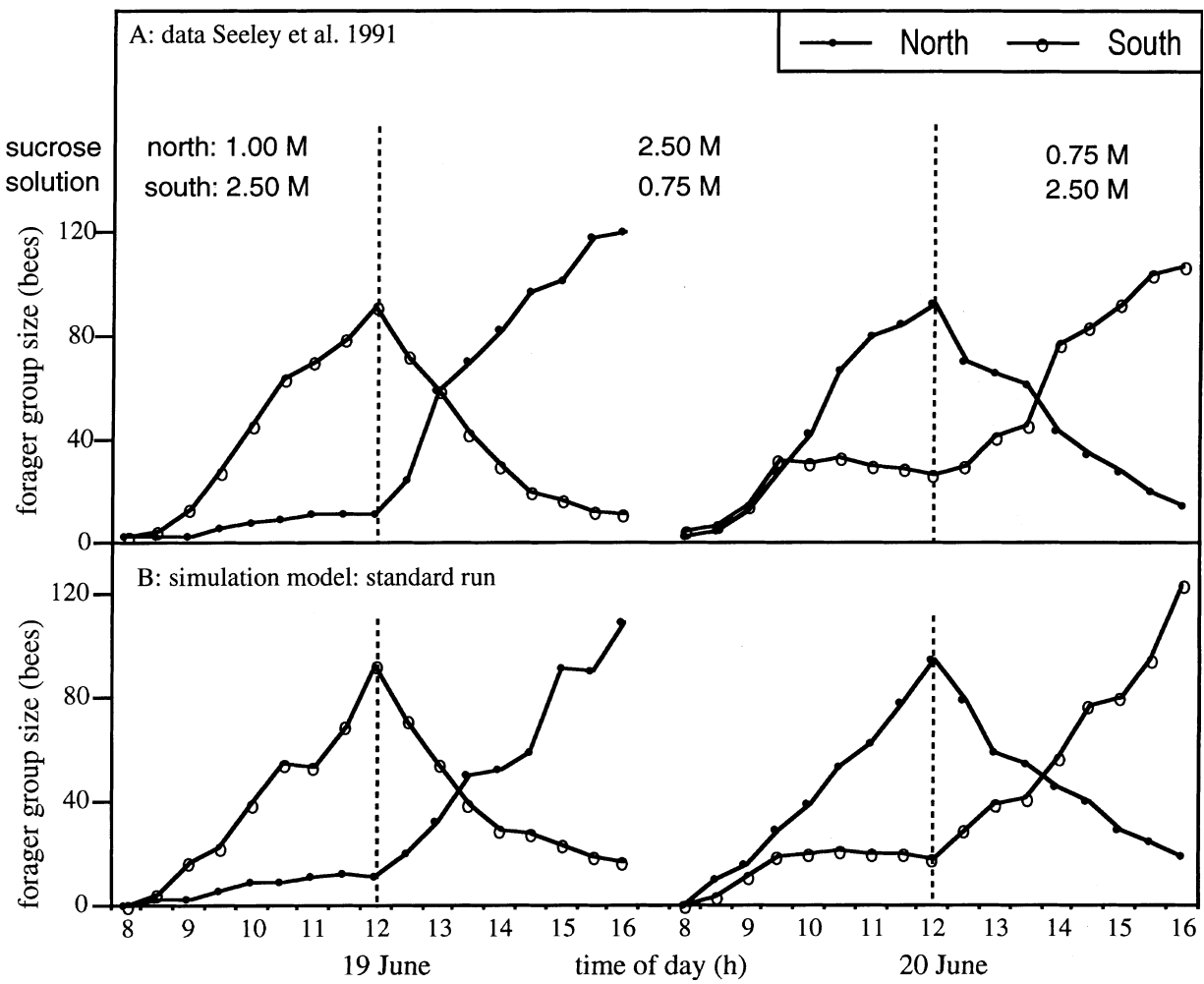




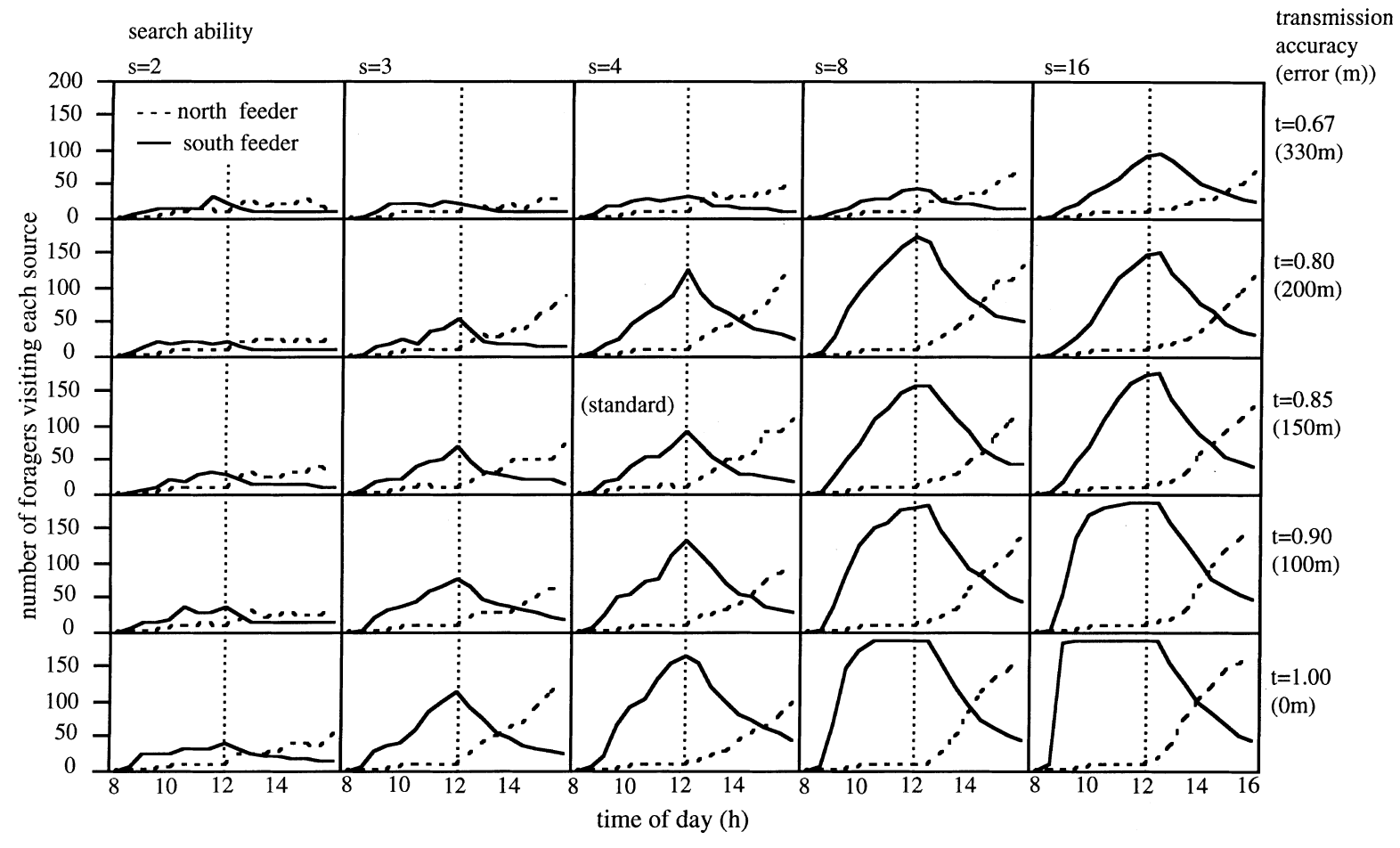

Fig. 4 One-day simulations for 25 combinations of transmission accuracy $(t=0.67,0.8,0.85,0.9,1.0)$ and search ability $(s=2,3,4$, $8,16)$. Abandoning probability was 0.07 in all runs. The $y$-axis gives the total number of different foragers that visited the sources per half hour. The north feeder (dashed lines) contained $1.00 \mathrm{M}$ before 1200 hours and $2.5 \mathrm{M}$ thereafter; the south feeder (solid lines) contained $2.5 \mathrm{M}$ before 1200 hours and $0.75 \mathrm{M}$ thereafter. The graph in the middle is the one with the standard setting of the parameters. These results show that the precision with which the source position is communicated and the ability to search the source are both important factors. Adaptive allocation to the two sources occurs within the whole range of parameter values

vising the model did not turn out to be so easy. At first, the simulated forager allocation pattern on the first and on the second day did not agree with the observed pattern shown in Fig. 3A. The set of individual behavioural rules we initially devised, mainly on the basis of the empirical regularities described by Seeley in his papers and in his recent book (1995), turned out to be insufficient to explain the observed forager allocation pattern. We first explain how we extended the set of rules to obtain a satisfactory agreement between simulated and observed forager pattern on the first day. Then, using the one-day model, we vary three bee-related factors (transmission accuracy, search ability and abandoning probability) in order to understand how the forager pattern depends on these factors. Next, we explain how we extended the model with an extra rule and an extra memory capacity for the bees so that we obtained a satisfactory agreement between simulated and observed forager pattern on the second day. Using this two-day simulation model, we vary two other factors, one sourcerelated factor (the profitabilities of the two sources) and one colony-related factor (the size of the worker force) in order to assess the effect of these factors on the forager allocation pattern.

\section{Model 1: simulating one day}

\section{Fitting the model to Seeley et al.'s empirical data}

Our first model was used to simulate one day with the two sources switched once at 1200 hours. Although the forager patterns generated by this model resembled the empirical data, there was a peculiar discrepancy between our model outcome and Seeley et al.'s observations: in the first half hour after the switch, there was no decrease in the number of foragers visiting the south feeder in the model. This was a direct consequence of the rule we initially used in our model: as soon as a bee detects a source she will find this source without any extra search effort the next time she flies out from the hive to the source. This means that all bees which visited the rich south feeder in the half hour before the switch will definitely also visit this source in the half hour directly after the switch. Since this however was not observed by Seeley et al. (see Fig. 3A, 19 June), clearly something had to be wrong with our rule. We decided to make the chance of finding a source that had been visited before dependent on the search ability of the bee and on the number of visits already paid to this source. The standard search ability was chosen such that the chance of finding the same source increases from about 25 to $100 \%$ in three successive flights after the first detection of the source. When this rule was incorporated in the model, many of the bees which visited the south feeder just for the first, second or third time in the half 
hour before the switch did not find it again in the following half hour. The simulated pattern of forager allocation on the first day is now in good agreement with the observed pattern (see first day in Fig. 3A, B). We discuss below alternative and additional factors that could be responsible for the observed decrease in the number of visiting bees immediately after a source concentration has been switched from high to low.

\section{The forager pattern at different parameter settings}

First, 25 simulations with different values of transmission accuracy and search ability were run, all with the abandoning probability set to the standard value of 0.07 , and a total forager workforce of 200 bees (Fig. 4). These results show that the precision with which the source position is communicated has a clear influence on the number of bees that visit the sources. Except when the search ability is very low $(s=2)$, we see that the better the position of the source is communicated, the more foragers visit the two sources. As expected, the ability to search the source also turns out to be important. The better the search ability of the bees, the more foragers will visit the two sources. If the circumstances are such that the memorised source position is subject to intense random error (due to some internal or external randomly disturbing influences), then obviously the forager workforce is not really suited to its task. But even with a very weak search ability, the foragers are still adaptively allocated to the two sources.

Second, we varied the abandoning probability for the $0.75 \mathrm{M}$ source between 0.00 and 0.20 per flight trip (with standard settings of $t=0.85$ and $s=4$ ). If the abandoning probability is zero, the colony does not allocate many foragers to the rich source after the source concentrations have been switched. Since none of the bees that have exploited the rich south feeder in the morning are inclined to follow recruitment dances, the only bees that can be recruited for the north feeder in the afternoon are the bees that are still naive and the ones that cannot locate the south feeder again after one or a very few visits. So the rise in the number of foragers exploiting the north feeder in the afternoon is critically dependent on the total size of the workforce. In the current model, the size of the workforce is fixed. If there would be an influx of naive young bees into the workforce as well as an outflux of old experienced bees, the adaptability of the colony would probably be stronger than it was in the case under study.

With the abandoning probability set to a high value, many foragers are available to be recruited for the north feeder after the 1200 hours switch. On the other hand, with a (very) high abandoning probability, the 12 bees which had experience with the north feeder at the start of the run might abandon this source before 1200 hours. To prevent this abandonment, Seeley et al. (1991) in their experiment supplied the north feeder with a $1.0 \mathrm{M}$ rather than a $0.75 \mathrm{M}$ sugar solution in the morning. We did the same in our simulation runs. Moreover, we made the abandoning tendency of each bee dependent on both the concentration of the source just visited as well as on the value of the abandoning probability parameter. The abandoning probability for the $1.0 \mathrm{M}$ source is always set to a value that is 0.15 lower than the value for the $0.75 \mathrm{M}$ source. Now if the abandoning probability parameter is set to a high value, a proportion of the 12 bees which initially had experience with the north feeder will abandon this source before 1200 hours. This effect is visible in the bottom graph of Fig. 5 where the abandoning probability for a $0.75 \mathrm{M}$ source is set to 0.20 , and thus to 0.05 for a $1.0 \mathrm{M}$ source. Almost all bees abandon the $1.0 \mathrm{M}$ north feeder before 1200 hours and the result is a very slow recruitment to the north feeder in the afternoon. If the abandoning probability is still higher, there are no bees left with information about the north feeder. In summary, the probability of abandoning a poor source should neither be too low nor too high for adaptive allocation to take place.

Model 2: simulating two consecutive days

\section{Fitting the model to Seeley et al.'s empirical data}

When we used the same model as above to simulate two consecutive days, the foraging pattern of the second day

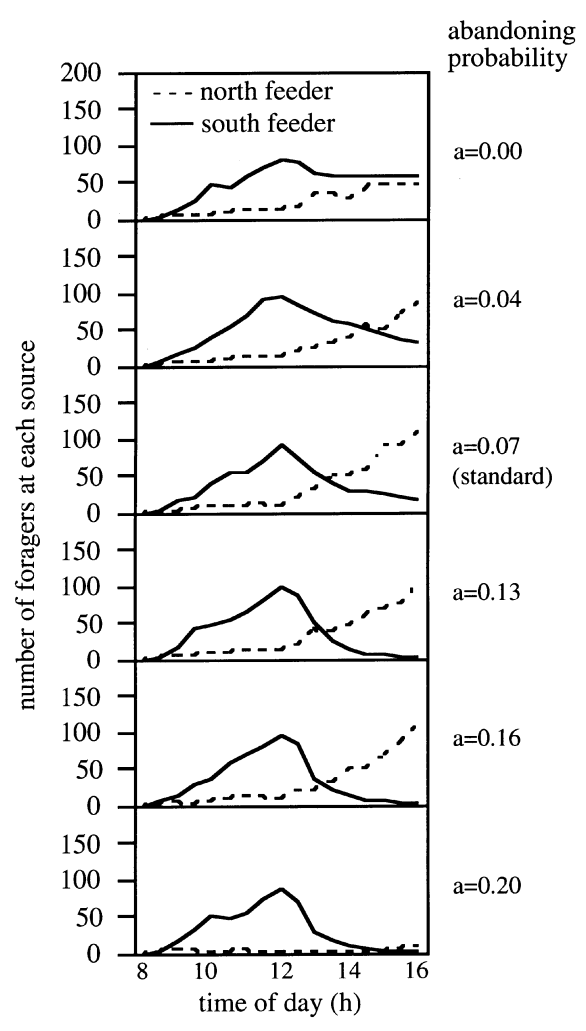

Fig. 5 Simulated one-day forager patterns resulting from simulation runs with different values for the abandoning probability $(0.0,0.04$, $0.07,0.13,0.20)$. Transmission accuracy and search ability have standard values of 0.85 and 4 , respectively. These results show that for adaptive allocation to take place, the probability of abandoning a poor source should neither be too low nor too high 
did not agree with Seeley et al.'s observations; in particular, the number of foragers visiting the south feeder in the morning of the second day did not increase (compare Fig. 3A). It was clear right away that the foraging pattern on the second day depends crucially on the experience the bees have accumulated on the previous day. But exactly what information obtained by a bee on one day is carried over to the next? In the basic model, the bee remembers only the source position of the last source she has visited. This however resulted in a forager pattern in which the number of foragers visiting the poorer source on the second day did not increase. First, we slightly extended the memory of a bee: instead of remembering only the position and profitability of the last source found she could also remember the time of day at which she first found this source (Bogdany 1978). Additionally, we included an extra rule, namely that a bee spontaneously makes a reconnaissance flight to her remembered source at or right after the time of day she first fed on this source the day before. To our surprise, this extension still did not give rise to the required increase in the number of visitors to the south feeder on day 2 . The reason is that a south-source forager is bound to be recruited away to the rich north feeder on the second morning (because the low profitability associated with the memory of the south feeder makes her receptive to follow dances) before the time has come for her to make a reconnaissance flight to her own south feeder. Therefore we decided to further increase the memory capacity of the bee. Besides remembering the last source found, a forager now also memorises the highest profitability of this source and the time of day this source was found. In other words, the bees that visited the rich south feeder in the morning of the first day started the next morning with the memory that that source was a rich one thereby overruling the memory that the same source was found to be poor in the afternoon. When we incorporated this in our model and also the extra rule that a bee spontaneously makes a reconnaissance flight to this most profitable source at or shortly after the time of day she first fed on this source the day before, a good fit was obtained between the simulated and observed foraging pattern on the second day (see Fig. 3). [As an aside it should be pointed out that the slow increase in the number of foragers visiting the 1.0 M north source on the first morning (Fig. 3B) is a consequence of this rule and of the extra assumption that the times of day memorised by the 12 bees with initial experience on the north feeder are randomly distributed between 0800 and 1100 hours.] The existence of such an extra memory capacity seems very plausible, since experiments by Bogdany (1978) have shown that honeybees can link time of day, colour and scent together in their memory (see also Gould 1993). Inclusion of the extra behavioural rule is supported by the fact that there are numerous observations of bees making reconnaissance flights in the morning (e.g. Seeley 1983, 1995; Seeley et al. 1991; von Frisch 1967).

Note that the increase in the number of foragers at the north feeder on the second morning and the increase at the south feeder (according to the current model rules) have different causes. Only the 12 originally experienced foragers make reconnaissance flights in the morning to the north feeder. The increase in the number of foragers is due mainly to reactivated foragers which have visited the north feeder only in the afternoon when it was rich (and therefore have a memorised time of day later than 1200 hours), and which now only need to follow a dance for a short time to find out that this rich source is again providing nectar, thereby confirming their knowledge.

\section{Varying the profitability regime}

Using this two-day model we made two other runs, one with the highest sugar concentration set to $1.5 \mathrm{M}$ instead of $2.5 \mathrm{M}$, and another run with the lowest concentration set to $1.5 \mathrm{M}$ instead of $0.75 \mathrm{M}$. It turns out that the level of the profitabilities of the two sources as well as the absolute difference between them are important factors. If the highest sugar concentration is set to $1.5 \mathrm{M}$ instead of $2.5 \mathrm{M}$, a picture is obtained that is qualitatively similar to the standard picture, but with much lower numbers of foragers visiting and exploiting the two sources (see Fig. 6a).

According to the observations presented by Seeley et al. (1991, Table 1), the abandoning probability for a $1.5 \mathrm{M}$ sugar solution is zero (the probability of continuing to visit the source is 1). Given this fact (which we included into our model), the forager pattern obtained with the second run in which the lowest sugar concentration is set to $1.5 \mathrm{M}$ instead of $0.75 \mathrm{M}$ (and the highest concentration remains $2.5 \mathrm{M}$ ) is readily understandable. In the afternoon of the first day, the bees keep on visiting the $1.5 \mathrm{M}$ south source, and similarly the bees keep on visiting the $1.5 \mathrm{M}$ north source in the afternoon of the second day (see Fig. 6b). Recruitment in the first afternoon to the $2.5 \mathrm{M}$ north source is not very strong because many bees continue visiting the $1.5 \mathrm{M}$ south source and the pool from which inexperienced bees can be recruited is not very large, since the total foraging workforce $(N)$ has been set to the standard value of 200 bees in this simulation run.

\section{Varying the foraging workforce}

Next we made a series of runs in which the total size of the worker force $(N)$ was varied (Fig. 7). Using the standard setting of values for the other parameters, we obtained five graphs of forager patterns which are qualitatively similar, except possibly for the simulation run with only 50 bees. When the size of the worker force ranges between 100 and 250 bees, we see that adaptive allocation of the foragers to the two sources occurs consistently. So, adaptive allocation appears to be a rather robust process. The forager patterns only differ in height, which is a direct consequence of the differences in total foraging workforce. 


\section{Discussion}

In several steps, a model was developed that reproduces the forager pattern observed in an experiment by Seeley et al. (1991). A good fit between observed and simulated collective forager patterns was obtained when the model included rules in which the bees (1) relied on the information acquired from previous flights to a source (e.g. profitability and time of day when the source was found), (2) used positional information obtained by attending recruitment dances and (3) did not abandon a (temporarily) deteriorated source too fast or too slowly.

Validating the model: the link between observation and modelling

If some part of the model output does not correspond to real-world observations, something is clearly wrong with or missing from the model. This is not to say, however, that agreement of the model output (the colony foraging pattern) with reality guarantees the validity of the model. Qualitatively similar colony forager patterns can be the result of quite different sets of individual behaviour rules. Therefore, to construct a valid model and thereby obtain the correct explanation for the observed patterns of collective foraging, it is necessary that each and every rule in the model expressing an empirical regularity is verified by means of empirical investigations. In the development of the current model, the

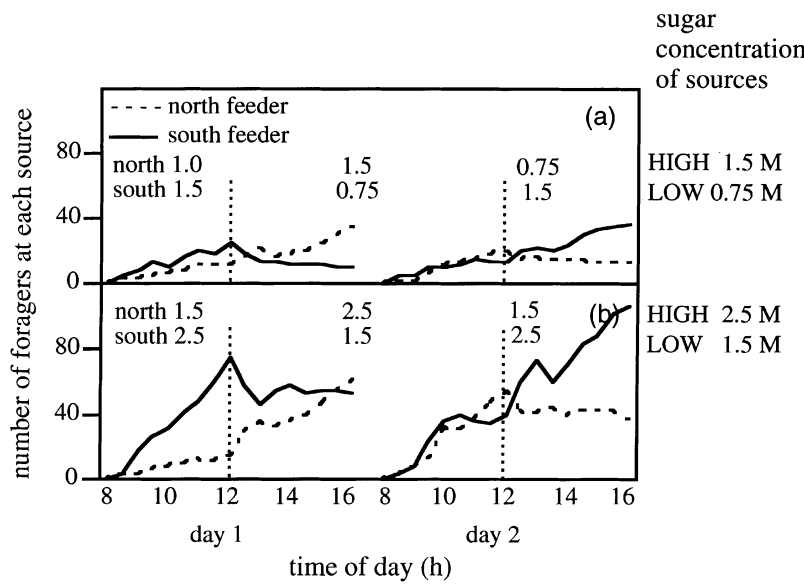

Fig. 6 Two simulated two-day forager patterns: with highest source profitability set to $1.5 \mathrm{M}$ instead of $2.5 \mathrm{M}$ (a) and with lowest source profitability set to $1.5 \mathrm{M}$ instead of $0.75 \mathrm{M}(\mathbf{b})$. In both runs, the total foraging workforce is set to the standard value of 200 bees. The outcome of the first run shows that adaptive allocation takes place, but with lower numbers of visiting foragers. The second run shows that in the afternoon of the first day, adaptive allocation does not fully occur. Recruitment to the $2.5 \mathrm{M}$ north source is rather slow compared to the strong increase found in reality and in the standard simulation run (Fig. 3A, B). This is due to the fact that many of the foragers stay with the $1.5 \mathrm{M}$ south source, and since the total workforce comprises 200 bees, there are not so many inexperienced bees left to be recruited to the $2.5 \mathrm{M}$ north source following specific questions cropped up. These questions have to be answered in future empirical research.

Why do fewer bees visit the south feeder in the first half hour after the switch (1200-1230 hours) than during the half hour before the switch?

One possible explanation is that a bee which has visited a source only once is not capable of finding the same source again on her next flight to the source. It might take several flights before she is able to fly to the source straightaway. This memory-based search behavior has been incorporated in our model by means of the search ability parameter. If the search ability (or rather the 'refind' ability) is low, many not-so-experienced recruits which found the south source only for the first or second time in the half hour before the switch do not succeed in finding it again in the half hour after the switch. Only the fully experienced bees, that is, those bees that have visited this source more than three times already, will definitely visit it again right after the switch. If, on the other

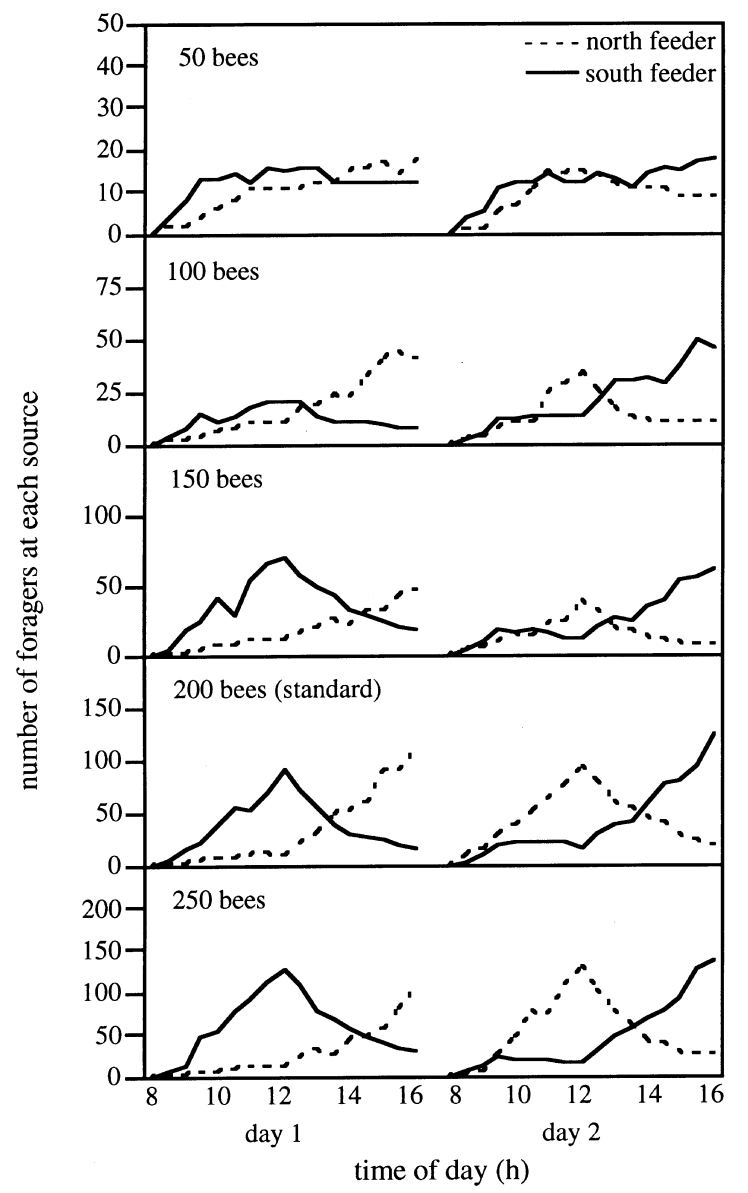

Fig. 7 Simulated two-day forager patterns with different values for the size of the forager workforce $(50,100,150,200,250)$. These results show that adaptive allocation is a rather robust process. Only with a very low workforce of 50 bees does adaptive allocation not really occur 
hand, bees are able to remember a source perfectly well after visiting the source only once, then all bees visiting the source before the switch will also visit this source directly after the switch. In that case, the number of visitors directly after the switch would be at least as high as just before the switch. This effect can be seen in the forager patterns that were obtained with simulation runs in which the search ability was set to the maximum value of 16 (see the five rightmost graphs in Fig. 4).

Other factors might also cause a decrease in the number of foragers visiting the source directly after the switch. In particular, the number of bees present at the source or flying to the source might be a cue for the less experienced bees that helps them find the source again (Friesen 1973). Observations on Melipona fasciata (Biesmeijer and Vork 1997) suggest that a number of bees do not land on the source because they have already perceived the deterioration of the source from cues in the neighbourhood. The cue might be a visual one, e.g. they might observe from a short distance that no or only few bees are present at the source (bees that $d o$ visit the deteriorated source only stay for a very short time). An olfactory cue might also play a role. Bees that have visited the deteriorated source could have marked the source by means of a repellent scent to prevent others from revisiting the source (Giurfa 1993). In Giurfa's experiment, the bees landed on the feeder with repellent scent but immediately left again. To explain Seeley's observations on the basis of this behavior, the foraging bees would have to smell this repellent scent at a short distance from the source; otherwise they would still be counted as visitors. It is likely that the more experienced bees react more rapidly to such cues and are the first to abandon the feeder.

Finally, the observed decrease in number of visiting foragers might be due to a difficulty for the observer to record the exact number of visitors (T.D. Seeley, personal communication). After the switch, bees stay only for a very short time on the source. They land on it, inspect it, and then keep on flying around for a while in the close vicinity of the source. Because of the very short visiting time of the bees and the general turmoil of flying bees, observers may have been unable to record the exact number of bees that visited the source.

What causes the increase in the number of bees visiting the rich north feeder in the morning of the second day?

It could be that these bees are reactivated bees, that is they are experienced bees which are informed that their north source is providing nectar again simply by following only a few waggle runs of a dance that advertises the rich north source. Alternatively (or additionally) these bees could be making independent reconnaissance flights to their known source. If that were the case, this would mean that bees do not have to memorise the earliest time of day at which a source was found. Instead, they simply require some cue which announces that the working day has started; this instigates all bees to make a reconnaissance flight to their known sources (rich or not). In honey-bees both possibilities seem to work simultaneously (von Frisch 1923). The shaking signal (or vibration dance) performed in the morning appears to function as a trigger for other bees with a slumbering tendency to start foraging or increase their foraging-related activities (Schneider et al. 1986a, b; Nieh 1998; Seeley et al. 1998).

What causes the increase in the number of bees visiting the poor south feeder in the morning of the second day?

The most plausible cause (which we incorporated in our model) is that the bees that had visited the south feeder during the morning of the day before are making independent reconnaissance flights. Since the south feeder has a low profitability, bees returning from these flights perform hardly any dances, and therefore there will be no or possibly only a few reactivated foragers for this source. Reconnaissance flights of experienced bees have been reported by von Frisch (1923).

Another possible cause (not incorporated in our model) might be that the experienced bees with a knowledge of the south feeder are triggered to make a reconnaissance flight to their source by the anise scent that is brought into the hive by the bees returning from the north feeder, which are the ones that perform the most and the longest dances. In the original experiment, both feeders contained the scent anise in a vented reservoir beneath the feeder and also in the sucrose solution (Seeley et al. 1991, p. 278).

\section{Is the colony's adaptability due to the adaptability of the bees?}

The colony as a whole appears to adapt itself quickly to a new situation in the environment. This adaptability of the colony can be the result of the adaptabilities of the individual bees: depending on the strength of the abandoning probability, a bee will sooner or later be open for new information after her known source has deteriorated. This has been implemented in our current model in accordance with the observations and model presented in Seeley et al. (1991).

The same adaptability of the colony could, however, also result when the bees are much less reluctant to abandon (completely forget) their known source and when at the same time there is a high turnover rate of foraging bees. If there is a high outflux of experienced bees (for instance, due to death by predators and/or exhaustion, or simply because they stop exploiting their deteriorated source without becoming interested in following dances again), which is balanced by an influx of naive foragers from the pool of other (non-forager) bees, the adaptability of the colony's behavior is still guaranteed without the necessity for each bee to be flexible. 
From observing foraging histories of individual bees in detail, it appears that this is indeed the case in M. beecheii and M. fasciata bees (Biesmeijer and Ermers 1997; Biesmeijer and Tóth, in press). It might also play a role in Apis mellifera.

How do honey-bee recruits search for the indicated source?

Besides these four specific empirical questions that cropped up while we were developing our model, a more general problem came to the fore. While experimenting with our model, we realised that a major feature was missing from it: namely, how is a bee's searching (and also refinding) behaviour in the field regulated? In our present model, the search flights of recruits are controlled by the transmission accuracy and search ability parameters which determine, respectively, the random error in the communicated source position and the randomness of the search (depending on how well the source position is memorised). The transmission accuracy parameter in our model is based on the research done by Towne and Gould (1988) on the spatial precision of the honey-bees' dance communication. They have shown that the distance and directional errors made by recruited bees searching for different targets between 100 and $700 \mathrm{~m}$ from the hive are similar in magnitude. Their measurements also "show that the angular information transmitted by the waggle dances of A. mellifera improves rapidly with increasing distance from the target. One likely proximate cause of this pattern involves the so-called dance divergence, meaning the systematic angular 'errors' in the direction indications given by dancers. The divergence angle, like the recruits' angular errors, decreases with increasing distance from the target" (Towne and Gould 1988, p. 132). However, it is not known for certain that a large divergence angle in the waggle dance does indeed cause recruit bees to make errors (Towne and Gould 1988, p. 151). Recently, Weidenmüller and Seeley (1997) have found that the divergence in dances for a nest site (which is always a point-like target) is about half that of dances for an equidistant feeder (which generally occurs in nature as a more or less spread-out patch of nectar sources). This shows that the "error" in the communicated direction of the target is indeed adaptively tuned to the type of target. In what way this divergence angle is used by the recruits in their search for the target is not known. The recruits' scatter around the target may be due not only to errors in the communicated target position but also to the way in which the recruits use this memorised target position during their search. To allow for this possibility, we introduced the search ability parameter. However, we did not introduce any rules specifying how the searching bees use cues present in the field, such as scents emanating from the two feeders (Seeley et al. 1991, p. 278), possible scents emanating from the body parts of the regular foragers, or simply the presence of other bees near or at the source. Such precise individual search rules were not necessary to obtain a good fit between observed and simulated forager pattern. This, of course, does not rule out the possibility that bees use such rules. The good fit is, most probably, a consequence of the specificity of the experimental design: only two sources with a very strict alternation of profitabilities. To model a wider range of situations (more sources with a greater variability of profitabilities in space and time), one needs knowledge about the search dynamics of the bees in the field. When investigating the foraging behaviour of individual bees, it is very difficult to obtain exact information on how the search flights of forager bees are influenced by the various visual, olfactory and other possible cues in the field and to find out to what extent the bees depend on the internal information stored in their neural system. It is evident that, in her search for the advertised source, a recruited bee uses both odour (e.g. Friesen 1973) and positional information (e.g. Michelsen et al. 1992), which she has acquired by following the waggle dance and has stored in her memory. The question is not whether honey-bees use either odour or positional information extracted from the waggle dance (Wenner and Wells 1990), but when and how they use these two kinds of information, as well as other possible kinds of information, for instance the presence of other bees near or at the source.

The attempt to develop a valid i-o model forces the modeller to evaluate the sufficiency and necessity of all these different behavioral possibilities and prevents the investigator from being biased in favour of one particular set of behavioral rules. In trying to construct a valid i-o model, one realises that it is essential to know what features in the environment can be perceived by the animal and what cues are acted upon that depend on her acquired experience.

\section{Features of i-o modelling}

The advantages of $\mathrm{i}-\mathrm{o}$ modelling have been extensively described (Huston et al. 1988; Hogeweg and Hesper 1990; Villa 1992; Judson 1994). Here we note only some of the features of $\mathrm{i}-\mathrm{o}$ modelling which we found particularly helpful. In the first place, developing an i-o model helps in formulating the individual behavioral rules. One is more or less forced to try to understand the bee's behaviour from the bee's point of view. Knowledge of how the individual behaviour of each bee is regulated by internal and external informational cues and stimuli then helps to explain how the global spatiotemporal patterns emerge from the behaviour of the locally interacting bees. I-o modelling allows for a stronger coupling between the two phases in scientific research: obtaining empirical data and constructing an explanatory theoretical model. It helps to determine the essential factors and reveals which empirical data are still missing. Since i-o modelling is a relatively easy way of investi- 
gating the global consequences of different individual rules or capacities, it can be helpful for discovering plausible behavioral rules.

The advantage of i-o modelling over differentialequation modelling is that in the former subtle but potentially important factors are less easily overlooked. In contrast to a differential-equations model, an i-o model explicitly incorporates several essential real-world aspects. For instance, the obvious but very relevant fact that at each moment in time each entity has a specific location in space and therefore each individual has at each moment a specific perceptual field is explicitly modelled. I-o models also allow individuals to have a memory. This makes it possible to investigate the relevance of distinguishing between experienced and naive bees and, more generally, to assess the role that memory, learning and information processing play in the collective foraging behaviour of the colony.

\section{The concept of nectar-foraging history}

Only after we had captured the refined concepts of scout, recruit, employed and unemployed forager, experienced and naive forager, and reactivated forager, were we able to construct a model in which it became clear that the foraging histories of the individual bees have a strong effect on the dynamics of the collective foraging behavior.

In the i-o model presented here, the concept of a behaving individual represented by a behaviour control structure is essential. The principles (rules) that regulate the nectar foraging history of a bee are instantiated in the behavior control structure (BCS) as depicted in Fig. 2. A characteristic of the BCS as presented here is that it is, as far as possible, based on empirically observed regularities. So, in a way, the particular BCS itself can be seen as an elaborated empirical concept, namely the concept of a nectar-foraging history of a forager bee. All possible individual foraging histories are implicit in this BCS and each particular foraging history can be derived from it. If certain bees show a foraging history that cannot be derived from this BCS, it means that this particular BCS is not a valid representation of a nectar forager.

This behaviour control structure can be extended in several ways. In the first place it could be extended to comprise not only the rules that control nectar-foraging behaviour, but other tasks, such as pollen foraging and food storing, as well. In principle, the whole life history of the individual bee could be represented by such a highly elaborated BCS. In this way, a concept of an organism as a life-long behaving individual is obtained (cf. Goodwin and Dawkins 1995). Next, internal (genetic, hormonal) and external (e.g. queen pheromone) factors could be added, which determine the parameter values in the life history BCS that control the transition from one task to the next, i.e. from one part of the BCS to another. The effects of these genetic, hormonal and external factors on the individual life histories and through these on the colony life history could then be studied.

\section{Future extensions}

The model presented in this paper faithfully simulates only a rather small range of collective foraging phenomena. The set of rules needs to be extended if it is to explain a larger range of empirical phenomena. The transition from the model that simulates one day to the model that simulates two consecutive days required an extension of the bee's memory capacities as well as an extra behavioural rule. Preliminary experience with situations involving more than two sources indicates that more detailed modelling of the searching behaviour is required to obtain simulation results which fit the observations in these cases. Of course, this is only to be expected: it is easier to model a very specific situation than a more general one. However, it is possible that the full set of rules necessary to explain most collective foraging phenomena in honey-bees is not very large. At the moment, however, there is a need for more empirical investigations into how the searching behaviour of the honey-bee is regulated. Unfortunately, not much empirical research has been done on this subject. How does a scout bee search for new sources? How does a newly recruited bee search for the source advertised in the waggle dance? A starting point can be found in the studies by Wenner et al. (1991, and references therein) and Friesen (1973).

The model could be extended still further. The assessment of the profitability of a nectar source by a bee could be made dependent on more factors than just the sugar concentration. A bee assesses the profitability of a nectar source by integrating information about variables such as nectar sweetness (sugar concentration), nectar abundance, nectar accessibility and distance from the hive (Seeley 1995, p. 118). Bees appear to measure the profitability of a source in terms of net energetic efficiency, that is (gain-cost)/cost. Also, the immediate past experience of a forager with a source whose profitability differs from that of the currently visited one affects her dancing behaviour (Raveret Richter and Waddington 1993). Additionally, the probability of dancing after returning from a source appears to be inversely related to the scent level in the sugar solution (Wells and Wenner 1971). These variables must be included in the model if the situation to be simulated is more complex than the current experiment in which the distances from the hive, the accessibilities, the abundances and the scent levels of the two sources were kept constant.

The model could also be extended with regard to the rules that regulate the behaviour of the bees inside the colony in relation to the number and/or the behaviour of food-storer bees in the hive which receive the collected nectar from the returning foragers. The model bee could be given an extra property in the form of a threshold for 
dancing, which regulates the motivation to perform a waggle dance after returning from a source. This threshold for dancing is the level of nectar source profitability above which the returning bee is motivated to dance. Each bee then has a different threshold for dancing which is adjusted in response to the search time needed to find a food-storer bee (Seeley 1995). The longer a nectar forager has to search for a receiver, the more she raises her dance threshold, that is, the less likely she will start a waggle dance. This search time is a reliable indicator of the current colony's nectar influx (Seeley and Tovey 1994; Seeley 1995, p. 121). When the model is thus extended it can simulate faithfully the phenomenon of cross-inhibition between forager groups (Seeley 1995, p. 142). So, clearly this dance threshold is a necessary factor if more complex collective foraging phenomena are to be explained. Another interesting feature that could be included in the model is the finding that returning forager bees perform tremble dances. These tremble dances, which are performed throughout the hive (Seeley 1992), serve to stimulate additional bees to function as nectar receivers (Seeley et al. 1996). If the search time for a nectar receiver becomes greater than about $40 \mathrm{~s}$, the probability that the nectar forager will perform a tremble dance increases quickly, at least if the profitability of her known source is high enough (Seeley 1992; Kirchner and Lindauer 1994). It has also been shown that tremble dances inhibit waggle-dancing bees to continue with this activity (Kirchner 1993; Nieh 1993). Another important behavior regulating the activity level of all kinds of behaviors and in particular foraging-related behaviors is the vibration dance or shaking signal (Schneider et al. 1986a,b; Nieh 1998; Seeley et al. 1998). This signal plays a role in arousing workers to greater activity. For instance, after a period of dearth, the first foragers returning from a rich source that has been empty for some days produce shaking signals to activate other workers to join in the foraging activities (Seeley et al. 1998). Foraging-age workers are triggered by the vibration dance to enter the waggle dance area of the hive (Schneider et al. 1986a). It has also been suggested that shaking functions as a signal indicating the beginning and close of the foraging "work day" depending on the time of day that food is available in the field (Nieh 1998).

If the model were to be extended in these ways it will be possible to (1) simulate a series of days to assess more fully the impact of previous foraging experience, (2) study the influence that patchiness and variation of sources in space and time have on the collective foraging patterns and the colony's foraging success and (3) study the way in which the availability of bees for performing foraging and other tasks within the nest (such as nectar receiving and storing) influence the collective foraging pattern.

It is well known that different species of social bees (honey-bees, stingless bees, bumblebees) show different collective foraging patterns. A future goal could be to find out to what degree these differences can be ex- plained by differences in their environments or by differences in their behavioural rules. That is, an interesting question is to investigate to what extent different species of bees have different behaviour control structures (Biesmeijer and Ermers 1997; Biesmeijer and Vork 1997; Biesmeijer et al. 1998).

Acknowledgements We are very grateful to Lada Lhotka for allowing us to use his programming environment for i-o modelling, HOBO. We thank two anonymous referees and Eltica de Jager Meezenbroek for their comments on the paper.

\section{References}

Bartholdi JJ, Seeley TD, Tovey GA, Vate JH van de (1993) The pattern and effectiveness of forager allocation among flower patches by honey bee colonies. J Theor Biol 160:23-40

Biesmeijer JC, Ermers M (1997) Social foraging in stingless bees: how Melipona fasciata chooses between nectar sources. In Biesmeijer: The organization of foraging in stingless bees of the genus Melipona: an individual-oriented approach. PhD thesis, Utrecht University, pp 147-170

Biesmeijer JC, Tóth E (in press) Individual foraging, activity level and longevity in the stingless bee Melipona beecheii in Costa Rica. Insect Soc

Biesmeijer JC, Vork W (1997) Flower constancy in stingless bees: the effect of patch position, odour and the presence of other bees on individual decision-making by Melipona fasciata foragers. In Biesmeijer: The organization of foraging in stingless bees of the genus Melipona: an individual-oriented approach. PhD thesis, Utrecht University, pp 171-188

Biesmeijer JC, Nieuwstadt MGL van, Lukács S, Sommeijer MJ (1998) The role of internal and external information in foraging decisions of Melipona workers (Hymenoptera: Meliponinae). Behav Ecol Sociobiol 42:107-116

Bogdany FJ (1978) Linking of learning signals in honeybee orientation. Behav Ecol Sociobiol 3:323-336

Camazine S, Sneyd J (1991) A model of collective nectar source selection by honey bees: self-organization through simple rules. J Theor Biol 149:547-571

Friesen LJ (1973) The search dynamics of recruited honey bees, Apis mellifera ligustica Spinola. Biol Bull 144:107-131

Frisch K von (1923) Über die "Sprache" der Bienen, eine tierpsychologische Untersuchung. Zool Jahrb 40:1-186

Frisch K von (1967) The dance language and orientation of bees. Harvard University Press, Cambridge, Mass

Giurfa M (1993) The repellent scent-mark of the honeybee Apis mellifera ligustica and its role as communication cue during foraging. Insect Soc 40:59-67

Goodwin B, Dawkins R (1995) What is an organism? A discussion. In: Thompson NS (ed) Perspectives in ethology, vol 11, pp $47-$ 60. Plenum, New York

Gould JL (1993) Ethological and comparative perspectives on honey bee learning. In: Papaj DR, Lewis AC (eds) Insect learning: ecological and evolutionary perspectives Chapman and Hall, New York, pp 18-50

Gould JL, Gould CG (1988) The honey bee. Freeman, New York Hogeweg P, Hesper B (1990) Individual-oriented modelling in ecology. Math Comput Model 13:83-90

Huston MA, DeAngelis D, Post W (1988) New computer models unify ecological theory. Bioscience 38:682-691

Judson OP (1994) The rise of the individual-based model in ecology. Trends Ecol Evol 9:9-14

Kirchner WH (1993) Vibrational signals in the tremble dance of the honeybee, Apis mellifera. Behav Ecol Sociobiol 33:169-172

Kirchner WH, Lindauer M (1994) The causes of the tremble dance of the honeybee, Apis mellifera. Behav Ecol Sociobiol 35:303308 
Lhotka L (1994) Implementation of individual-oriented models in aquatic ecology. Ecol Model 74:47-62

Michelsen A, Towne WF, Kirchner WH, Kryger P (1987) The acoustic near field of a dancing honey bee. J Comp Physiol A 161:633-643

Michelsen A, Andersen BB, Storm J, Kirchner WH, Lindauer M (1992) How honeybees perceive communication dances, studied by means of a mechanical model. Behav Ecol Sociobiol 30:143150

Nieh JC (1993) The stop signal of honey bees: reconsidering its message. Behav Ecol Sociobiol 33:51-56

Nieh JC (1998) The honey bee shaking signal: function and design of a modulatory communication signal. Behav Ecol Sociobiol 42:23-36

Raveret Richter MR, Waddington KD (1993) Past foraging experience influences honey bee dance behaviour. Anim Behav 46:123-128

Schneider SS, Stamps JA, Gary NE (1986a) The vibration dance of the honey bee. I. Communication regulating foraging on two time scales. Anim Behav 34:377-385

Schneider SS, Stamps JA, Gary NE (1986b) The vibration dance of the honey bee. II. The effects of foraging success on daily patterns of vibration activity. Anim Behav 34:386-391

Seeley TD (1983) Division of labor between scouts and recruits in honeybee foraging. Behav Ecol Sociobiol 12:253-259

Seeley TD (1992) The tremble dance of the honey bee: message and meanings. Behav Ecol Sociobiol 31:375-383

Seeley TD (1994) Honey bee foragers as sensory units of their colonies. Behav Ecol Sociobiol 34:51-62

Seeley TD (1995) The wisdom of the hive. Harvard University Press, Cambridge

Seeley TD, Tovey CA (1994) Why search time to find a food-storer bee accurately indicates the relative rates of nectar collecting and nectar processing in honey bee colonies. Anim Behav 47:311-316

Seeley TD, Towne WF (1992) Tactics of dance choice in honey bees: do foragers compare dances? Behav Ecol Sociobiol 30:5969

Seeley TD, Camazine S, Sneyd J (1991) Collective decision-making in honey bees: how colonies choose among nectar sources. Behav Ecol Sociobiol 28:277-290

Seeley TD, Kühnholz S, Weidenmüller A (1996) The honey bee's tremble dance stimulates additional bees to function as nectar receivers. Behav Ecol Sociobiol 39:419-427

Seeley TD, Weidenmüller A, Kühnholz S (1998) The shaking signal of the honey bee informs workers to prepare for greater activity. Ethology 104:10-26

Towne WF, Gould JL (1988) The spatial precision of the honey bees' dance communication. J Insect Behav 1:129-155

Villa F (1992) New computer architectures as tools for ecological thought. Trends Ecol Evol 7:179-183

Waddington KD, Kirchner WH (1992) Acoustical and behavioral correlates of profitability of food sources in honey bee round dances. Ethology 92:1-6

Weidenmüller A, Seeley TD (1997) Adaptive imprecision in waggle dances for nearby food sources? Adv Ethol 32:114

Wells PH, Wenner AM (1971) The influence of food scent on behavior of foraging honey bees. Physiol Zool 44:191-209

Wenner AM, Wells PH (1990) Anatomy of a controversy. Columbia University Press New York

Wenner AM, Meade DE, Friesen LJ (1991) Recruitment, search behavior, and flight ranges of honey bees. Am Zool 31:768-778

Communicated by R.F.A. Moritz 